\title{
Hierarchical Attribute-Guided Symmetric Diffeomorphic Registration for MR Brain Images
}

\author{
Guorong Wu, Minjeong Kim, Qian Wang, and Dinggang Shen \\ Department of Radioloy and BRIC, Univerity of North Carolina at Chapel Hill \\ \{grwu,mjkim, dgshen\}@med.unc.edu, qianwang@email.unc.edu
}

\begin{abstract}
Deformable registration has been widely used in neuroscience studies for spatial normalization of brain images onto a standard space. Due to high anatomical variances across individual brains, registration performance could be limited when trying to estimate entire deformation pathway either from template to subject or subject to template. Symmetric image registration offers an effective way to simultaneously deform template and subject images towards each other until they meet at the middle point. Although some intensity-based registration algorithms have nicely incorporated this concept of symmetric deformation, the intensity matching between two images may not necessarily imply the correct matching of anatomical correspondences. In this paper, we integrate both strategies of hierarchical attribute matching and symmetric diffeomorphic deformation for building a new symmetric-diffeomorphic registration algorithm for MR brain images. The performance of our proposed method has been extensively evaluated and further compared with top-ranked image registration methods (SyN and diffeomorphic Demons) on brain MR images. In all experiments, our registration method achieves the best registration performance, compared to all other state-of-the-art registration methods.
\end{abstract}

\section{Introduction}

Modern medical imaging technology provides a convenient way to perform clinical diagnosis and research, such as human brain development, aging, and disease-related abnormalities. In order to measure subtle anatomical or functional difference, accurate deformable registration plays an important role in dealing with confounding intrasubject variability in the longitudinal studies and inter-subject variability in the crosssectional studies.

A lot of deformable image registration methods have been proposed in the last decades. A comprehensive survey can be found in [1]. In general, the goal of deformable registration is to estimate the deformation field from template (fixed image) to subject (moving image) by maximizing a certain image similarity measurement between template and warped subject image. As a highly ill-posed problem, regularizing the deformation field is necessary for resolving the uncertainty in correspondence detection during the registration.

Recently, 14 deformable registration methods have been comprehensively evaluated in [2] based on the registration performance on human brain MR images. 
Interestingly, all of these 14 registration methods are intensity-based. However, the use of image intensity only is not sufficient to deal with the confounding inter-subject variations, due to the lack of specific features to establish reasonable anatomical correspondences. In [2], SyN [3] is recommended as the top deformable registration method, which uses the concept of symmetric registration by estimating the deformation pathway from two ends (template and subject) to the middle point.

Inspired by this symmetric registration mechanism [3], we propose a feature-based symmetric registration method in this paper with three novel strategies. First, we define attribute vectors for detecting anatomical correspondences and key points with distinctive image feature for hierarchically driving the entire registration procedure in image registration. Specifically, the attribute vector on each voxel considers not only local image appearance but also edge information. The key points are randomly sampled in the non-uniform and hierarchical fashion, assuring that they have distinctive features and cover the entire brain. Second, robust correspondence matching (by comparing attribute vectors) is performed in a symmetric way. We simultaneously optimize the deformations on the key points from both template and subject until they meet at the middle point of the whole deformation pathway (between template and subject). Since the correspondence matching is always performed between the key points on the deformed template and the deformed subject in the progress of image registration, the correspondence matching is relatively easier, compared to the case of directly finding correspondences between the original template and subject images. Hence, our method is able to handle large anatomical variations among individual brains under registration. Third, we adapt the registration procedure to a space of diffeomorphic transformation by following the efficient log-domain approach in [4]. Therefore, the estimated deformation field by our registration method is smooth and invertible, but the computation time can be greatly reduced, compared with other complicated diffeomorphic registration methods [3].

To compare the registration performance of our proposed registration method, we choose two state-of-the-art registration methods, i.e., SyN [3] and diffeomorphic Demons [4], as the references, since they achieve the top-ranked performance as evaluated in [2] and are widely used in neuroscience study. The registration accuracy is comprehensively evaluated on real human brain MR images (elderly brains aged from 65-85, LONI LBPA40 [5], and NIREP NA0 [6] datasets), all with manually-labeled ROIs. In all experiments, our proposed feature-based registration method achieves the best registration performance in terms of registration accuracy.

\section{Methods}

The goal of deformable registration is to find a transformation $F=\{f(x) \mid f(x)=x+$ $\left.h(x), x \in \mathfrak{R}^{3}\right\}$ between template $T$ and subject $S$, with displacement $h(x)$ defining the mapping of the point coordinates $\Omega_{T}$ of template $T$ to $\Omega_{\mathrm{S}}$ of subject $S$. Here, $\Omega_{T}, \Omega_{S} \subset \Re^{3}$. In this section, we will first present the overview of our registration method in Section 2.1. The energy function and its solution will be explained in Section 2.2 and Section 2.3, respectively. 


\subsection{Overview of Our Symmetric Feature-Based Registration Method}

The overview of our symmetric feature-based registration method is shown in Fig. 1. Instead of estimating the deformation field $F$ from template to subject, we estimate two deformation fields starting from template and subject, respectively. Thus, we can obtain the deformation fields $\phi_{1}$ from template space and $\phi_{2}$ from the subject. Both $\phi_{1}$ and $\phi_{2}$ point towards the common space in the middle of the pathway between template and subject. In the end, the deformation field from template to subject can be calculated by $F=\phi_{1} \circ \phi_{2}^{-1}$, where ' $\circ$ ' denotes the composition of two deformation fields. Similarly, the inverse deformation field $F^{-1}$ from subject to template can be obtained by $F^{-1}=\phi_{2} \circ \phi_{1}^{-1}$.

As mentioned earlier, anatomical correspondence is very important in deformable image registration. Therefore, we define attribute vector $\vec{a}$ on each voxel to establish the correspondence. To further improve the correspondence detection, we hierarchically select the key points (located at the salient regions in the MR brain images) to steer the deformation of registration, as well as to guide the deformation of other lessdistinctive points. As displayed in Fig. 1(a), only a small number of points located at the critical areas of brain image are selected as the key points (red points denote for key points of template and blue points for key points of subject) to drive the image registration. With the progress of registration as in the bottom of Fig. 1(a), more and more voxels are qualified as the key points to refine the deformation field, as it can seen in the bottom of Fig. 1(a) that $\phi_{1}$ and $\phi_{2}$ change from global deformation in the beginning to local deformation in the end of registration. The definition of attribute vector and hierarchical key point selection will be explained next.

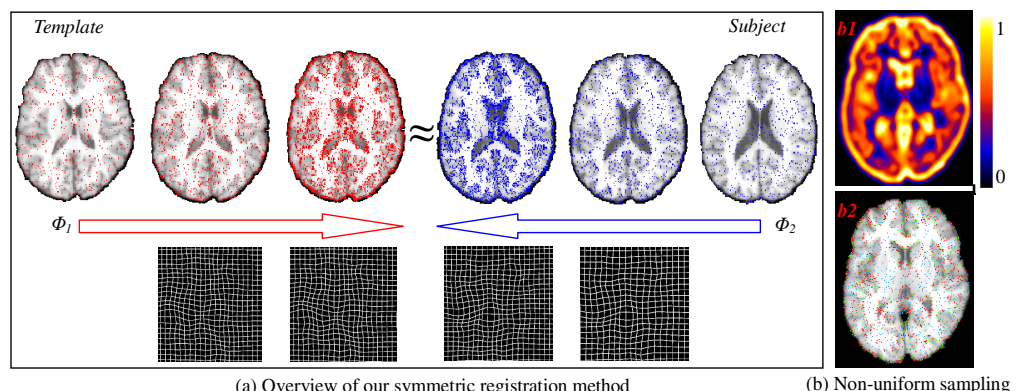

Fig. 1. The framework of our symmetric feature-based registration algorithm (a) and the key modules in our method, i.e., key point selection by non-uniform sampling (b).

\subsection{Energy Function in Our Symmetric Registration Method}

In our symmetric registration method, both template and subject will deform toward the middle point iteratively, as shown in Fig. 1. (a). Thus, we use $T^{k}=T\left(\phi_{1}^{k}\right)$ and $S^{k}=S\left(\phi_{2}^{k}\right)$ to denote the tentatively-warped template and subject images w.r.t. the deformation fields $\phi_{1}^{k}$ and $\phi_{2}^{k}$ in the end of the $k^{t h}$ iteration, respectively.

Attribute Vector: Attribute vector is used as the morphological signature to characterize geometric information around each voxel. Although some rich image descriptors, e.g., 
SIFT and SURF, work well on correspondence matching, they are computational expensive and sensitive to the structural variations across individual brain images. For robust correspondence matching, we incorporate both local image appearance and edge information in our registration method by arranging all image intensities and gradients in a neighborhood (with radius $r$, e.g., $r=3 \mathrm{~mm}$ ) into the attribute vector $\vec{a}$. Normalized cross correlation is used as the similarity measure to evaluate the similarity between point $x$ in the deformed template $T^{k}$ and point $y$ in the deformed subject $S^{k}$, which is denoted as $\operatorname{NCC}\left(\vec{a}_{T}^{k}(x), \vec{a}_{S}^{k}(y)\right)$. Furthermore, we define the feature discrepancy as $\eta\left(\vec{a}_{T}^{k}(x), \vec{a}_{S}^{k}(y)\right)=\frac{1-N C C\left(\vec{a}_{T}^{k}(x), \vec{a}_{S}^{k}(y)\right)}{2}$, which ranges from 0 to 1 . Apparently, we evaluate the similarity not only on the single pair of points $x$ and $y$, but also on their respective sub-volumes. Since we explicitly detect correspondences only on the key points, not every voxel in the image, our registration method is efficient, although the computation of normalized cross correlation is more expensive than simple intensity difference.

Key Points Selection: Here, we use an importance sampling strategy to hierarchically select the key points in the image. Specifically, we first smooth, and normalize the gradient magnitude values in the whole image domain. Then, these values are roughly considered as the importance of each voxel during the registration. It is worth noting that the gradient magnitude is selected as the importance of each voxel mainly because of its low computational cost, although other advanced methods can be also applied here. Based on the importance map, a set of key points can be sampled, with higher importance value indicating the higher likelihood of the underlying location being selected in the non-uniform sampling. Fig. 1 shows the non-uniform sampling based on the importance map (Fig. 1(b1)). The initial subset of key points and also the key points selected in the later two stages of registration are displayed in blue, green, and red in Fig. 1(b2), respectively. It is clear from Fig. 1(b2) that the key points are more concentrated at areas with rich edge information, where the importance values are high. After we apply the non-uniform sampling to both $T^{k}$ and $S^{k}$, we can obtain the template key points $X^{k}=\left\{x_{i}^{k} \mid i=1, \ldots, M_{T}^{k}\right\}$ and the subject key points $Y^{k}=\left\{y_{i}^{k} \mid i=1, \ldots, M_{S}^{k}\right\}$ at the $k^{\text {th }}$ iteration of registration, where $M_{T}^{k}$ and $M_{S}^{k}$ are the numbers of key points in the deformed template and deformed subject in the $k^{t h}$ iteration.

Energy Function: In $(k+1)^{\text {th }}$ iteration, we will estimate the incremental deformation $\varphi_{1}^{k+1}$ from $T^{k}$ to $S^{k}$ and $\varphi_{2}^{k+1}$ from $S^{k}$ to $T^{k}$. As we will explain later, the refined deformation fields $\phi_{1}^{k+1}$ and $\phi_{2}^{k+1}$ in the end of the $(k+1)^{t h}$ iteration can be obtained by integrating the incremental deformation fields $\varphi_{1}^{k+1}$ and $\varphi_{2}^{k+1}$.

Given the currently estimated incremental deformation $\varphi_{1}^{k+1}\left(x_{i}^{k}\right)$ at key point $x_{i}^{k}$, exhaustive search is performed to refine the correspondence w.r.t. each candidate point $u$ in a search neighborhood $n\left(\varphi_{1}^{k+1}\left(x_{i}^{k}\right)\right)$, according to the two criteria: 1$)$ the feature discrepancy should be as small as possible between $\vec{a}_{T}^{k}\left(x_{i}^{k}\right) \in \Omega_{T^{k}}$ and $\vec{a}_{S}^{k}(u) \in \Omega_{S^{k}}$; 2) the spatial distance between candidate point $u$ and the tentatively estimated location $\varphi_{1}^{k+1}\left(x_{i}^{k}\right)$, i.e., $\left\|\varphi_{1}^{k+1}\left(x_{i}^{k}\right)-u\right\|^{2}$, should be as close as possible.

Since there are a lot of uncertainties in correspondence matching, encouraging multiple correspondences is proven effective to alleviate the ambiguity issue. For a particular template key point $x_{i}^{k}$, a probability $\pi_{i}^{u}$ (called as spatial assignment) is 
assigned to each candidate point $u \in n\left(\varphi_{1}^{k+1}\left(x_{i}^{k}\right)\right)$ during correspondence matching. For robust correspondence matching, the candidate points even with large discrepancy still might have the chance to contribute to the correspondence matching in the beginning of registration. As the registration progresses, only the candidates with the most similar attribute vectors will be considered until the exact one-to-one correspondence is allowed in the end of registration for achieving the registration specificity. This dynamic procedure can be encoded with the entropy term on the probability, i.e., $\pi_{i}^{u} \cdot \log \pi_{i}^{u}$. Here, high degree of entropy implies the fuzzy assignment while low degree means almost binary matching. We use a scalar value $t^{k+1}$ to act as the temperature to enforce the dynamic change on correspondence assignment.

The optimization of incremental deformation field $\varphi_{2}^{k+1}$ follows the same way as discussed above, except we use $\tau_{j}^{v}$ to denote the assignment of each candidate point $v$ in the search neighborhood $n\left(\varphi_{2}^{k+1}\left(y_{j}^{k}\right)\right)$. Thus, the total energy function in estimating incremental deformation field $\varphi_{1}^{k+1}$ and $\varphi_{2}^{k+1}$ is given as:

$$
\begin{aligned}
E\left(\varphi_{1}^{k+1}, \varphi_{2}^{k+1}\right) & =\sum_{i=1}^{M_{T}^{k}} \sum_{u \in n\left(\varphi_{1}^{k+1}\left(x_{i}^{k}\right)\right)}\left\{\pi_{i}^{u} \cdot\left[\eta\left(\vec{a}_{T}^{k}\left(x_{i}^{k}\right), \vec{a}_{S}^{k}(u)\right)+\left\|\varphi_{1}^{k+1}\left(x_{i}^{k}\right)-u\right\|^{2}\right]+t^{k+1} \cdot\left(\pi_{i}^{u} \cdot \log \pi_{i}^{u}\right)\right\} \\
& +\sum_{j=1}^{M_{S}^{k}} \sum_{v \in n\left(\varphi_{2}^{k+1}\left(y_{j}^{k}\right)\right)}\left\{\tau_{j}^{v} \cdot\left[\eta\left(\vec{a}_{T}^{k}(v), \vec{a}_{S}^{k}\left(y_{j}^{P}\right)\right)+\left\|\varphi_{2}^{k+1}\left(y_{j}^{k}\right)-v\right\|^{2}\right]+t^{k+1} \cdot\left(\tau_{j}^{v} \cdot \log \tau_{j}^{v}\right)\right\} \\
& +\beta \cdot\left\{B\left(\varphi_{1}^{k+1}\right)+B\left(\varphi_{2}^{k+1}\right)\right\},
\end{aligned}
$$

where $B($.$) measures the bending energy of incremental deformation fields [7]. \beta$ in Eq. 1 is a scalar value to control the strength on deformation smoothness.

\subsection{Optimization of Symmetric Deformation Pathways}

First, the spatial assignment $\pi_{i}^{v}$ can be calculated by minimizing $E$ in Eq. 1 w.r.t. $\pi_{i}^{u}$ :

$$
\pi_{i}^{u}=\exp \left\{-\frac{\eta\left(\vec{a}_{T}^{k}\left(x_{i}^{k}\right), \vec{a}_{S}^{k}(u)\right)+\left\|\varphi_{1}^{k+1}\left(x_{i}^{k}\right)-u\right\|^{2}}{t^{k+1}}\right\} .
$$

Similarly, the spatial assignment $\tau_{j}^{v}$ can be obtained as:

$$
\tau_{j}^{v}=\exp \left\{-\frac{\eta\left(\vec{a}_{T}^{k}(v), \vec{a}_{S}^{k}\left(y_{j}^{p}\right)\right)+\left\|\varphi_{2}^{k+1}\left(y_{j}^{k}\right)-v\right\|^{2}}{t^{k+1}}\right\} .
$$

It is clear that spatial assignment $\pi_{i}^{u}$ and $\tau_{j}^{v}$ are penalized in the exponential way according to the discrepancy degree $\eta$. Notice that the temperature $t^{k+1}$ is the denominator of the exponential function in Eqs. 2 and 3. Therefore, when $t^{k+1}$ is very high in the beginning of registration, even though the discrepancy $\eta$ might be large or the candidate location is far away, the candidate point still might have the chance to be selected as the correspondence. As registration progresses, the specificity of correspondence will be encouraged by gradually decreasing the temperature $t^{k+1}$ to a small degree, until only the candidate point with the smallest discrepancy being selected as the correspondence in the end of registration. 
After obtaining $\pi_{i}^{u}$ for each candidate $u$, the estimated incremental deformation on key point $x_{i}^{k}$ can be computed by optimizing energy function $E$ in Eq. 1 w.r.t. $\varphi_{1}^{k+1}$ :

$$
\hat{\varphi}_{1}^{k+1}\left(x_{i}^{k}\right)=\sum_{u \in n\left(\varphi_{1}^{k+1}\left(x_{i}^{k}\right)\right)} \pi_{i}^{u} \cdot u / \sum_{u \in n\left(\varphi_{1}^{k+1}\left(x_{i}^{k}\right)\right)} \pi_{i}^{u} .
$$

Similarly, the incremental deformation of each $y_{j}^{k}$ can be updated as:

$$
\hat{\varphi}_{2}^{k+1}\left(y_{j}^{k}\right)=\sum_{v \in n\left(\varphi_{2}^{k+1}\left(y_{j}^{k}\right)\right)} \tau_{j}^{v} \cdot v / \sum_{v \in n\left(\varphi_{2}^{k+1}\left(y_{j}^{k}\right)\right)} \tau_{j}^{v} .
$$

The estimated incremental deformation fields $\hat{\varphi}_{1}^{k+1}$ and $\hat{\varphi}_{2}^{k+1}$ are sparse which have the displacements only on the key points. Then TPS [7] is used to immediately interpolate the dense deformation fields $\hat{\varphi}_{1}^{k+1}$ and $\hat{\varphi}_{2}^{k+1}$ with the bending energy minimized. In order to ensure the invertibility of output deformation fields, we follow an efficient non-parametric diffeomorphic approach [4] to adapt the optimization of $\phi_{1}^{k+1}$ and $\phi_{2}^{k+1}$ to the space of diffeomorphic transformation. The basic idea is to consider the incremental deformation fields $\hat{\varphi}_{1}^{k+1}$ and $\hat{\varphi}_{2}^{k+1}$ in the vector space of velocity fields and then map them to the space of diffeomorphism through the exponentials, i.e., $\exp \left(\hat{\varphi}_{1}^{k+1}\right)$ or $\exp \left(\hat{\varphi}_{2}^{k+1}\right)$.

Specifically, the following steps will be applied to calculate the deformation field $\hat{\phi}_{1}^{k+1}$ under the framework of diffeomorphism: 1) compute the exponential of incremental deformation field $\exp \left(\hat{\varphi}_{1}^{k+1}\right)$ by the scaling and squaring method [4]; 2) compose the exponential with the previously estimated deformation field by $\hat{\phi}_{1}^{k+1}=$ $\left.\phi_{1}^{k} \circ \exp \left(\hat{\varphi}_{1}^{k+1}\right) ; 3\right)$ the inverse deformation field can be computed by $\left(\hat{\phi}_{1}^{k+1}\right)^{-1}=$ $\left(\phi_{1}^{k}\right)^{-1} \circ \exp \left(-\hat{\varphi}_{1}^{k+1}\right)$. Similarly, the deformation pathway $\hat{\phi}_{2}^{k+1}$ and its inverse $\left(\hat{\phi}_{2}^{k+1}\right)^{-1}$ can be computed by performing these three steps.

Finally, the latest estimated deformation pathway $\hat{\phi}_{1}^{k+1}$ and $\hat{\phi}_{2}^{k+1}$ will be used to deform the original template and subject images, and obtain $T^{s+1}$ and $S^{k+1}$ for the next iteration. The whole procedure (i.e., estimating the incremental deformation fields $\left(\varphi_{1}^{k+1}\right.$ and $\left.\varphi_{2}^{k+1}\right)$ and updating the symmetric deformation pathways $\phi_{1}^{k+1}$ and $\phi_{2}^{k+1}$ ) will be repeated until the template and subject images meet at the middle point.

\section{Experiments}

To evaluate the registration performance, we test our symmetric feature-based registration method on elderly brain images, as well as NIREP-NA0 and LONI-LPBA40 datasets, by comparing it with the two state-of-the-art registration methods, i.e., SyN [3] and diffeomorphic Demons (D. Demons) [4], which demonstrate good performance in [2]. For SyN and diffeomorphic Demons registration methods, we use their best parameters as listed in the supplementary document of [2].

Registration Results on 18 Elderly Brains: In this experiment, 18 MR brain images of elderly subjects were used, each with image dimension $256 \times 256 \times 124$ and resolution $0.9375 \times 0.9375 \times 1.5 \mathrm{~mm}^{3}$. After selecting one subject as the template, we registered all other 17 subjects onto the template by SyN, diffeomorphic Demons, and our proposed method, respectively. The mean images (averaging up all registered 


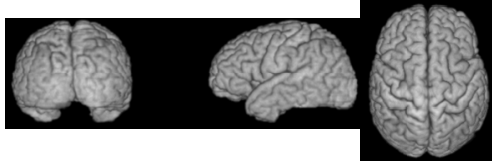

(a) Mean image by SyN

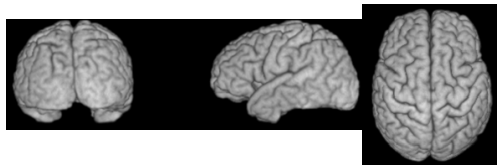

(c) Mean image by diffeomorphic demons

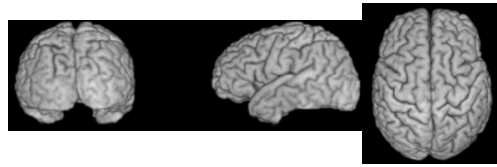

(e) Mean image by S-HAMMER

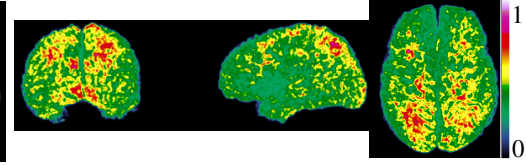

(b) Variance image by SyN

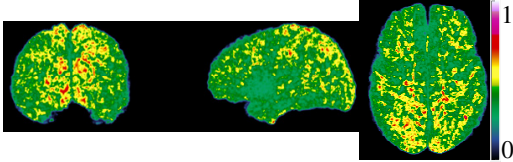

(d) Variance image by diffeomorphic demons

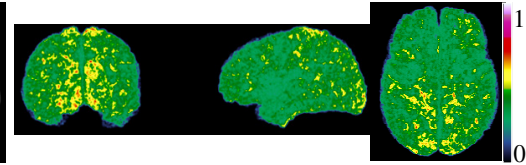

(f) Variance image by S-HAMMER

Fig. 2. Demonstration of registration performance on 18 elderly MR brain images. The mean images upon all registered images by SyN, diffeomorphic demons, and our method are shown in (a), (c), and (e), respectively. To examine the registration consistency across different subjects, the local entropy of tissue probability maps across all aligned images by SyN, diffeomorphic Demons, and our method are shown in (b), (d), and (f), respectively, indicating that our method has the least variation.

subject images) by SyN, diffeomorphic Demos, and our registration method are shown in Fig. 2(a), (c), and (e), respectively.

Since the images have been segmented into GM, WM, CSF and VN, we can calculate the voxel-wise overlap ratio between the warped subjects and the template (Table 1). Here, we set the target overlap ratio as the overlap measurement since it was used in [2] to evaluate all 14 registration methods. By masking out the background voxels, the averaged target overlap ratios between template and each aligned subject are $70.10 \%$ for SyN, $70.88 \%$ by deformorphic Demons, and $74.13 \%$ by our proposed method, respectively. For further quantification of registration consistency, we computed the voxel-by-voxel entropy of tissue probability maps across all aligned subjects. High entropy value indicates less consistency. For our proposed method, the averaged entropy is 0.40 , which is nearly $17 \%$ improvement compared to 0.48 obtained by deformorphic Demons, and $35 \%$ improvement compared to 0.62 obtained by SyN. The respective 3D map of entropy value is shown in Fig. 2 by SyN (2b), deformorphic Demons (2d), and our method (2f). It is worth noting that the improvement on tissue overlap ratios between our method and other two methods are significant in paired t-test $(p<0.05)$.

Table 1. Target overlap ratio of white matter, gray matter, ventricle on 18 elderly brains by SyN, diffeomorphic Demons (D. Demons), and our method. (unit: \%)

\begin{tabular}{lllll}
\hline & White matter & Gray matter & Ventricle & Overall \\
\hline SyN & $71.28 \pm 3.33$ & $56.63 \pm 3.58$ & $82.40 \pm 3.35$ & $70.10 \pm 3.42$ \\
D. Demons & $72.05 \pm 5.24$ & $58.22 \pm 8.00$ & $82.36 \pm 4.50$ & $70.88 \pm 6.34$ \\
Our method & $75.22 \pm 3.22$ & $62.36 \pm 3.92$ & $84.82 \pm 1.86$ & $74.13 \pm 2.86$ \\
\hline
\end{tabular}


NIREP NA0 Dataset: One of the 16 images is randomly selected as the template. Then we register the rest 15 images to the template image by $\mathrm{SyN}$, diffeomorphic Demons, and our proposed method. After registration, the target overlap ratio can be calculated for each of the 32 manually delineated labels. We repeat this procedure for 5 times by selecting different subject as template. The mean and standard deviation of target overlap ratios across all subjects and all ROIs are $60.25 \pm 1.67 \%$ by SyN, $64.23 \pm 1.38 \%$ by diffeomorphic Demons, and $65.17 \pm 1.16 \%$ by our registration method, respectively.

LONI LPBA40 Dataset: In this experiment, we use the LONI LPBA40 dataset [5] with 40 brain images and 54 manually labeled ROIs in each brain image. Similarly, we employ SyN, diffeomorphic Demons, and our method to align 39 brain images to a randomly selected template image. We repeat this procedure for 5 times as well. The overall tissue overlap ratio is $73.23 \pm 1.35 \%$ by $\mathrm{SyN}, 73.12 \pm 1.62 \%$ by diffeomorphic Demons, and $74.00 \pm 1.26 \%$ by our method, respectively.

\section{Conclusion}

In this paper, we proposed a new feature-based symmetric registration method for MR brain images. Compared with other intensity-based registration methods, our method achieves more accurate registration. Our symmetric feature-based registration method produces better registration results than the two top-ranked intensity-based registration methods (SyN and D. Demons) on all three experiments.

\section{References}

1. Maintz, J.B.A., Viergever, M.A.: A survey of medical image registration. Medical Image Analysis 2, 1-36 (1998)

2. Klein, A., Andersson, J., Ardekani, B.A., Ashburner, J., Avants, B., Chiang, M.-C., Christensen, G.E., Collins, D.L., Gee, J., Hellier, P., Song, J.H., Jenkinson, M., Lepage, C., Rueckert, D., Thompson, P., Vercauteren, T., Woods, R.P., Mann, J.J., Parsey, R.V.: Evaluation of 14 nonlinear deformation algorithms applied to human brain MRI registration. NeuroImage 46, 786-802 (2009)

3. Avants, B.B., Epstein, C.L., Grossman, M., Gee, J.C.: Symmetric diffeomorphic image registration with cross-correlation: Evaluating automated labeling of elderly and neurodegenerative brain. Medical Image Analysis 12, 26-41 (2008)

4. Vercauteren, T., Pennec, X., Perchant, A., Ayache, N.: Diffeomorphic demons: efficient non-parametric image registration. NeuroImage 45, S61-S72 (2009)

5. Shattuck, D.W., Mirza, M., Adisetiyo, V., Hojatkashani, C., Salamon, G., Narr, K.L., Poldrack, R.A., Bilder, R.M., Toga, A.W.: Construction of a 3D probabilistic atlas of human cortical structures. NeuroImage 39, 1064-1080 (2008)

6. Christensen, G.E., Geng, X., Kuhl, J.G., Bruss, J., Grabowski, T.J., Pirwani, I.A., Vannier, M.W., Allen, J.S., Damasio, H.: Introduction to the Non-rigid Image Registration Evaluation Project (NIREP). In: Pluim, J.P.W., Likar, B., Gerritsen, F.A. (eds.) WBIR 2006. LNCS, vol. 4057, pp. 128-135. Springer, Heidelberg (2006)

7. Bookstein, F.L.: Principal warps: thin-plate splines and the decomposition of deformations. IEEE Transactions on Pattern Analysis and Machine Intelligence 11, 567-585 (1989) 